\title{
Gadofosveset Trisodium
}

National Cancer Institute

\section{Source}

National Cancer Institute. Gadofosveset Trisodium. NCI Thesaurus. Code C81057.

The trisodium salt form of gadofosveset, an injectable, intravascular, amphiphilic gadolinium-based contrast agent (GBCA) used with magnetic resonance angiog raphy (MRA) imaging. Gadofosveset is a stable gadolinium diethylenetriaminepentaacetic acid (Gd-DTPA) chelate derivative with a diphenylcyclohexylphosphate group. Upon injection, gadofosveset binds reversibly to endogenous serum albumin which increases its intravascular retention time compared to non-protein binding contrast agents. The serum albumin binding also increases T1-relaxivity of gadofosveset. This produces an increase in signal intensity of blood, thereby enhancing the visualization of blood vessels upon MRA and may aid in the diagnosis of certain blood vessels and heart disorders. 